\title{
FAMILIARES E PESSOAS CONHECIDAS DE USUÁRIOS DE DROGAS ILÍCITAS: RECORTE DE OPINIÕES SOBRE LEIS E POLÍTICAS PÚBLICAS DE UMA COMUNIDADE DA ZONA OESTE DO RIO DE JANEIRO, BRASIL
}

\author{
Jaqueline da Silva ${ }^{1}$ \\ Bruna Brands ${ }^{2}$ \\ Edward Adlaf ${ }^{3}$ \\ Norman Giesbrecht ${ }^{3}$ \\ Laura Simich ${ }^{3}$
}

Maria da Gloria Miotto Wright ${ }^{4}$

Silva J, Brands B, Adlaf E, Giesbrecht N, Simich L, Wright MGM. Familiares e pessoas conhecidas de usuários de drogas ilícitas: recorte de opiniões sobre leis e políticas públicas de uma comunidade da Zona Oeste do Rio de Janeiro, Brasil. Rev Latino-am Enfermagem 2009 novembro-dezembro; 17(Esp.):803-9.

Este artigo é parte do estudo "Uso de drogas ilícitas em sete países latino-americanos e Canadá: perspectivas críticas de familiares e pessoas conhecidas" (7LACC) que investigou quatro domínios: fatores protetores e de risco, iniciativas de prevenção, unidades de tratamento e leis e políticas. Apresenta uma seção dos resultados baseados em quatro itens do domínio leis e políticas - como percebidas por familiares e pessoas conhecidas de usuários de drogas ilícitas, residentes na comunidade, recrutados em unidades urbanas de cuidados primários à saúde, localizadas na zona oeste da cidade do Rio de Janeiro. Trata-se de estudo corte temporal, multimétodos que entrevistou 100 adultos maiores de 18 anos, cognitivamente saudáveis. Resultados e conclusões chave foram o não atendimento dos princípios fundamentais da legislação do Sistema Único de Saúde (SUS)/Lei 8.080/90 e a equivocada aplicação das leis e políticas públicas sobre drogas ilícitas.

DESCRITORES: drogas ilícitas/legislação \& jurisprudência; leis; transtornos relacionados ao uso de substâncias/prevenção \& controle; familia

\section{FAMILY AND ACQUAINTANCES OF ILICIT DRUG USERS: COMMUNITY PERSPECTIVES ON LAWS AND PUBLIC POLICIES IN WESTERN RIO DE JANEIRO, BRAZIL}

This article is part of the study "Illicit Drug Use in Seven Latin American Countries and Canada: Critical Perspectives of Family and Familiars" (7LACC), which investigated four domains: protective and risk factors; preventive initiatives; treatment facilities; and laws and policies. The article presents a section of the results based on four items of the laws and policies domain - as perceived by the family and acquaintances of illicit drug users living in the community. Participants were recruited in urban primary health care units located in Western Rio de Janeiro (city), Brazil. This multi-method, crosstemporal study performed interviews with 100 adults (18 years of age or older), all cognitively healthy. Results and key conclusions included non-compliance with the fundamental principles of the Unique Health System Legislation / Law 8.080/ 90 and the erroneous implementation of laws and public policies on illicit drug.

DESCRIPTORS: street drugs/ legislation \& jurisprudence; laws; substance-related disorders/prevention \& control; family

\section{LA FAMILIA Y LAS PERSONAS CONOCIDAS DE USUARIOS DE DROGAS ILÍCITAS - OPINIONES SOBRE LEYES Y POLÍTICAS PÚBLICAS DE UNA COMUNIDAD EN LA ZONA OCCIDENTAL DE RIO DE JANEIRO, BRASIL}

Este articulo forma parte del estudio "Uso de Drogas Ilícitas en Siete Países Latino Americanos y Canadá - Las Perspectivas Críticas de la Familia y de Personas Conocidas" (7LACC). Fueron investigados cuatro dominios: factores protectores y de riesgo; iniciativas inhibidoras; facilidades de tratamiento; y, leyes y políticas. Se presenta una sección de resultados relacionados a leyes y políticas, describiendo como ellas fueron percibidas por familiares y personas cercanas a los usuarios de drogas ilícitas, que estaban inscritos en unidades primarias urbanas de asistencia a la salud, situadas en la zona occidental de la ciudad de Río de Janeiro, en Brasil. Se trata de una investigación transversal, que utilizó múltiples métodos, en la cual fueron entrevistados 100 adultos, que tenían más de 18 años de edad, sin problemas cognoscitivos. Los resultados y las principales conclusiones fueron el incumplimiento de los principios fundamentales del Sistema Unificado de Salud (SUS) / Ley 8.080/90 y la inadecuada implementación de leyes y políticas públicas relacionadas a las drogas ilícitas.

DESCRIPTORES: drogas ilícitas; leyes; prevención \& controle; familia

${ }^{1}$ Enfermeira, Pós-Doutora, Escola de Enfermagem Anna Nery, Universidade Federal do Rio de Janeiro, Brasil, e-mail: jackiedasilva@hotmail.com. ${ }^{2}$ Ph.D., Pesquisador Sênior, Office of Research and Surveillance, Drug Strategy and Controlled Substances Programme, Health Canada and Public Health and Regulatory Policies, Centre for Addiction and Mental Health, CAMH, University of Toronto, Canadá, e-mail: bruna_brands@camh.net. ${ }^{3}$ Ph.D., Pesquisador, Health Systems Research and Consulting Unit, Centre for Addiction and Mental Health, CAMH, University of Toronto, Canadá, e-mail: edward_adlaf@camh.net, norman_giesbrecht@camh.net, laura_simch@camh.net. ${ }^{4}$ Ph.D., Especialista Sênior, Coordenador, Educational Development Program, Demand Reduction Section, Inter-American Drug Abuse Control Commission, CICAD, Organization of American States, OAS, Estados Unidos, e-mail: gwright@oas.org. 


\section{INTRODUÇÃO}

O consumo de drogas ilícitas é assunto chave na agenda internacional, pois causa danos sociais e econômicos e afeta o desenvolvimento dos países. O problema das drogas ilícitas foi analisado em vários projetos e é objeto de diretrizes de ação(1) estabelecidas por organizações internacionais/ nacionais, não-governamentais e governamentais, nos níveis do governo federal, estadual e municipal, e em outras instituições e associações. Evidências encontradas em pesquisas ${ }^{(2-5)}$ descrevem a situação dos países no que diz respeito à estrutura institucional, redução de demanda, redução de provisão e medidas de controle, corroborando a ideia de que as drogas ilícitas representam tema internacional de grande relevância, bem como problema de saúde pública.

Em relação ao Brasil, o país é produtor ilícito de maconha e país de passagem de cocaína boliviana, colombiana e peruana, endereçadas à Europa e EUA $^{(6)}$. Para o Brasil, a maconha tem a prevalência mais alta $(1 \%)$, seguida pela cocaína $(0,4 \%)$ e anfetaminas $(0,3 \%)$. De acordo com o Escritório Nacional Antidrogas e a Organização das Nações Unidas, o Brasil é considerado país mediano em relação ao consumo de drogas ilícitas, com características e realidade bastante específicas, mas intrinsecamente relacionadas às de outros países, vizinhos e não vizinhos, influenciando com suas leis e políticas nacionais e sendo influenciado por leis e políticas internacionais ${ }^{(6)}$.

Para atingir, portanto, metas nacionais e internacionais para a redução da produção, provisão e demanda por drogas ilícitas é necessário levar em conta desafios regionais como a pobreza, desigualdades sociais, planejamento urbano precário, produção e tráfico de drogas ilícitas. Os desafios regionais latino-americanos incrementam os problemas relacionados às drogas e aumentam a necessidade de se compartilhar conhecimento, que deverá ser traduzido em prática mais efetiva.

Considerando que o conhecimento compartilhado é fruto de iniciativas coletivas envolvendo diferentes atores, apenas as perspectivas de políticos, cientistas e provedores de cuidado de saúde não são suficientes para lidar com os desafios vividos pelos usuários de drogas ilícitas e a comunidade. Por essa razão, perspectivas de comunidades com moradores usuários, seus familiares e/ou pessoas próximas - que vivem e dividem a experiência de sucessos e fracassos de iniciativas de prevenção, serviços de tratamento, aspectos legais - são críticas para a construção de conhecimento para desenho do perfil, acompanhamento e avaliação de ações e serviços dirigidos à comunidade.

O termo chave "comunidade", entretanto, tem sido usado na literatura, publicada entre 1990 e 2008, para descrever uma localidade passiva, em lugar de descrever agentes de informação ativos e críticos. Além disso, o conteúdo publicado durante o mesmo período está baseado em bancos de dados oficiais de órgãos internacionais e nacionais e com base em perspectivas políticas e de provedores de cuidado de saúde.

Nesse contexto, portanto, tornam-se necessárias duas perspectivas para preencher lacunas existentes no conhecimento: as perspectivas dos usuários de drogas ilícitas e as de seus familiares e pessoas próximas. Considerando a dificuldade de se proteger os informantes ao se recrutar usuários de drogas ilícitas para entrevistas, este estudo foi desenhado para construir conhecimento, investigando as perspectivas dos familiares e/ou pessoas próximas aos usuários de drogas ilícitas, em nove centros urbanos de sete países americanos latino-americanos e Canadá.

Levando em conta que a produção de conhecimento ocorre em uma via de mão dupla, o estudo multicêntrico foi dividido em duas fases. A fase um envolveu: (i) ouvir a opinião sobre experiências vividas, necessidades percebidas e demandas dos participantes, (ii) comparar e contrastar resultados com documentos oficiais, políticas, leis e normas. A fase dois continua a envolver o retorno: (i) apresentar resultados aos investigadores, profissionais de cuidado de saúde e gestores políticos para aumentar a conscientização para ações mais efetivas a curto, médio e longo prazo e (ii) oferecer recursos para a construção de meios mais efetivos de prevenção de saúde, bem como de intervenção e tratamento.

Por ser parte de uma pesquisa extensa, no entanto, o presente artigo trata de apresentar um recorte do quarto domínio, referente a leis e políticas sobre drogas no Brasil na perspectiva de residentes frequentadores de unidades básicas de saúde, na zona oeste da cidade do Rio de Janeiro.

\section{MÉTODO}

O estudo combinou dois métodos: o quantitativo e o qualitativo, com o objetivo de explorar 
os diferentes aspectos das experiências de vida dos participantes. A decisão de combinar os métodos quantitativo e qualitativo foi baseada no reconhecimento da equipe de que os métodos são complementares e possuem pontos fortes diferenciados ${ }^{(7)}$; ainda, a combinação de métodos oferece elementos para a discussão e análise dos $\operatorname{dados}^{(8)}$, permitindo a comparação de convergências e a confirmação dos resultados.

Para operacionalizar os estudos que combinam os dois métodos, a criação de conexões entre eles pode ser problema complexo no desenho da pesquisa. Então, como estratégia prática, o estudo de métodos combinados coletou, na seção quantitativa, informações demográficas e dados sobre os conhecimentos dos respondentes sobre fatores protetores e de risco, iniciativas de prevenção, serviços de tratamento, políticas e leis.

O estudo seguiu oito passos, compreendendo a coleta e análise das informações sobre as perspectivas dos participantes vinculadas às drogas ilícitas, fatores de risco e de proteção relacionados, iniciativas de prevenção, serviços de tratamento e aspectos legais: (i) descrição da realidade de cada centro urbano em relação a dados gerais e socioculturais, (ii) identificação de semelhanças e diferenças entre as realidades dos centros urbanos, (iii) obtenção de visão geral dos problemas das drogas em comunidade da zona oeste do Rio de Janeiro, (iv) descrição das perspectivas dos participantes de cada centro urbano, (v) identificação das semelhanças e diferenças entre as perspectivas dos participantes sobre a realidade da comunidade e centro urbano em questão, (vi) comparação das semelhanças e diferenças entre as perspectivas dos participantes nos 9 centros urbanos de 8 países (sete latino-americanos e Canadá), (vii) discussão dos achados da pesquisa, baseada na perspectiva de saúde internacional e (vii) elaboração de sugestões e recomendações, baseadas na análise dos resultados, considerando as especificidades de cada área, cidade e país participante.

O estudo teve início após a aprovação pelo Comitê de Ética e Pesquisa da Escola de Enfermagem Anna Nery (EEAN)/Hospital Escola São Fancisco de Assis (HESFA), sob Protocolo n.030/2007, datado de 25 de abril de 2007.

A pesquisadora principal selecionou o serviços/unidades-satélite básicas de saúde para o recrutamento. A técnica de bola de neve foi eleita como estratégia complementar de recrutamento, considerando-se a natureza ${ }^{(9)}$ delicada do tema drogas ilícitas, e provou ser mais eficaz do que os cartazes na comunidade-cenário em questão.

Os participantes foram incluídos no estudo após (i) atenderem os critérios específicos de inclusão e exclusão estabelecidos em perguntas de préseleção, (ii) tomarem conhecimento, por meio de descrição verbal, dos objetivos do estudo, procedimentos, do termo de consentimento livre e esclarecido e da segurança de confidencialidade, (iii) terem respondidas suas dúvidas sobre o estudo e os procedimentos de coleta de dados, (iv) consentirem em participar do estudo através da entrevista e (v) assinarem o formulário do termo de consentimento livre e esclarecido.

O número calculado de indivíduos que participariam do estudo multicêntrico foi de 1100 participantes, onde a pesquisadora principal responsável pelo cenário da zona oeste e sua equipe administraram 100 entrevistas quantitativas - a pessoas maiores de dezoito anos, autoidentificadas como cognitivamente preservadas. O trabalho em tela apresenta resultados de um recorte da base de dados da zona oeste. Importante destacar que, para reduzir a variabilidade, a pesquisadora principal recrutou os participantes na mesma instituição de saúde pública, de acordo com os critérios de inclusão e exclusão descritos na proposta do estudo.

O tamanho da amostra foi determinado com base nos recursos disponíveis em cada local e para garantir variação suficiente nas características e experiências dos pesquisadores participantes do estudo multicêntrico internacional. A análise em cada local de pesquisa incluiu a descrição das características, experiências e opiniões dos membros da família e pessoas próximas a usuários de drogas ilícitas. Os resultados obtidos receberam tratamento e análise estatística descritiva essencialmente com medidas de tendência central.

Os dados quantitativos foram coletados por meio de um instrumento com perguntas fechadas, com respostas documentadas em papel e formulários preenchidos pela pesquisadora principal e sua equipe de pesquisa. Explicações e dados adicionais foram incluídos na forma de diário de campo e mesmo para a obtenção de informações mais detalhadas sobre os objetivos do estudo ${ }^{(10)}$. Portanto, os dados coletados também incluíram notas de campo, com o registro pelos pesquisadores das impressões pessoais, dúvidas 
e interpretações preliminares observadas ou sentidas $^{(11-12)}$

\section{RESULTADOS}

Os 100 adultos participantes eram maiores de dezoito anos, cognitivamente preservados. Conforme identificado, $31(48,4 \%)$ das 64 mulheres e $25(68,9 \%)$ dos 36 homens declararam ser casados, $71 \%$ deles são cristãos, $37 \%$ deles são evangélicos e $34 \%$ são católicos, regularmente praticantes. Do total da amostra, $26 \%$ são atuantes membros de grupos comunitários, dentre os quais $4 \%$ em organizações de interesse, $10 \%$ em associações comunitárias e $12 \%$ outras, incluindo voluntariados e escolas de samba. O grau de escolaridade predominante na amostra foi de $49 \%$ com ensino fundamental incompleto, ocorrendo índice de 5\% de analfabetismo funcional, seguido por $20 \%$ com ensino médio incompleto e de $10 \%$ com ensino médio e formação técnica completos. A atividade de trabalho mais declarada foi "outra", de caráter informal, onde as especificações mais frequentes foram a confecção caseira de comestíveis, artesanato ou manufaturados e comercialização de produtos industrializados de perfumaria e maquiagem, através de revistas para pessoas de conhecidas (52\%), seguidas das atividades como dona de casa (18\%) e vendas diversas ou serviços (16\%). As situações de vínculo empregatício mais frequentes foram empregado (23\%), outra (22\%), autônomo (21\%), desempregado $(18 \%)$ e aposentado (14\%). Quanto às condições de moradia, embora residentes em área de baixa renda, $79 \%$ das pessoas entrevistadas residem em casa própria e apenas $12 \%$ em casa alugada. A maioria reside com filhos ( $51 \%)$, com cônjuge (44\%) e com outros familiares (38\%). As principais fontes de renda foram salários (30\%) e pensões, aposentadoria e anuidades $(19 \%)$ e os valores predominantes das rendas individual e familiar foram abaixo de $R \$$ $1.000,00 /$ mês e entre $R \$ 1.000,00$ - R\$2.999,00/mês, respectivamente. O acesso ao cuidado à saúde foi 92\% de caráter público (Sistema Único de SaúdeSUS) e $16 \%$ de caráter privado (Planos de Saúde), números que evidenciam a utilização de ambos os serviços de pelo menos $8 \%$ dos participantes.

$\mathrm{Na}$ Tabela 1 há indicação de que $46 \%$ dos participantes afirmaram que as leis e políticas sobre drogas ilícitas não garantem segurança e 71\% apontaram que as mesmas também não a beneficiam. No mesmo subitem, em relação aos usuários, baseados em suas experiências vividas ou observadas na comunidade, os entrevistados indicaram que as leis e políticas atuais não facilitam o tratamento e a recuperação dos usuários (79\%), não respeitam seus direitos humanos (73\%) e não os protegem (39\%) de alguma forma. Na verdade, de acordo com os depoimentos, muito frequentemente (74\%), as leis e políticas como estão, aumentam o comportamento criminal e, na grande maioria dos casos (78\%), também não diminuem as oportunidades de acesso às drogas ilícitas. No subitem sobre as leis serem punitivas aos usuários, as respostas ficaram mais equitativamente distribuídas, refletindo maior variação dentre as perspectivas.

Tabela 1 - Opinião sobre leis e políticas sobre drogas ilícitas no Brasil*

\begin{tabular}{|c|c|c|c|c|c|}
\hline Domínio: leis e políticas & $\begin{array}{c}\text { Concorda } \\
\text { totalmente }=1\end{array}$ & $\begin{array}{c}\text { Concorda } \\
\text { parcialmente }=2\end{array}$ & $\begin{array}{c}\text { Discorda } \\
\text { parcialmente }=3\end{array}$ & $\begin{array}{c}\text { Discorda } \\
\text { totalmente }=4\end{array}$ & Não sei $=8$ \\
\hline 1. Garantem a nossa segurança & 4 & 34 & 16 & 46 & 0 \\
\hline 2. Protegem o usuário de drogas & 6 & 6 & 18 & 39 & 3 \\
\hline 3. Punem o usuário de drogas & 34 & 37 & 34 & 19 & 1 \\
\hline 4. Punem os traficantes & 42 & 27 & 12 & 18 & 1 \\
\hline 5. Facilitam a reintegração social dos usuários & 11 & 3 & 8 & 76 & 2 \\
\hline 6. Facilitam o tratamento e a recuperação dos usuários & 4 & 8 & 7 & 79 & 2 \\
\hline 7. Beneficiam a sociedade & 3 & 11 & 12 & 71 & 3 \\
\hline 8. Respeitam os direitos humanos & 4 & 7 & 11 & 73 & 5 \\
\hline 9. Diminuem as oportunidades de se ter acesso às drogas & 2 & 5 & 14 & 78 & 1 \\
\hline 10. Aumentam o comportamento criminal & 74 & 6 & 4 & 13 & 3 \\
\hline
\end{tabular}

*Valores correspondentes à frequência total = percentual de respostas para cada item e respectivas opções.

A Tabela 2 estão apresentados os dados sobre as situações do envolvimento de pessoas em geral relacionadas com drogas ilícitas, as opiniões dos entrevistados de que não deveriam ser presas por consumo $(37 \%)$, mas deveriam ser presas em caso de tráfico $(82 \%)$, venda $(80 \%)$ e comportamentos 
violentos (57\%). Entretanto, os respondentes ficaram divididos no subitem 6 quanto à prisão ou não por porte de pequenas porções de droga, refletindo maior variação de opiniões.

Tabela 2 - Opinião sobre situações em que pessoas envolvidas com drogas ilícitas deveriam ser presas*

\begin{tabular}{|c|c|c|c|c|c|}
\hline Domínio: leis e políticas & $\begin{array}{c}\text { Concorda } \\
\text { totalmente = } 1\end{array}$ & $\begin{array}{c}\text { Concorda } \\
\text { parcialmente }=2\end{array}$ & $\begin{array}{c}\text { Discorda } \\
\text { parcialmente }=3\end{array}$ & $\begin{array}{c}\text { Discorda } \\
\text { totalmente }=4\end{array}$ & Não sei $=8$ \\
\hline 1. Usarem drogas ilícitas & 13 & 27 & 22 & 37 & 1 \\
\hline 2. Comprarem drogas ilícitas & 24 & 28 & 18 & 28 & 2 \\
\hline 3. Venderem drogas ilícitas & 80 & 10 & 0 & 8 & 2 \\
\hline 4. Traficarem drogas ilícitas & 82 & 10 & 0 & 7 & 1 \\
\hline 5. Terem comportamentos violentos & 57 & 11 & 7 & 24 & 1 \\
\hline 6. Carregarem pequenas porções de drogas & 20 & 34 & 22 & 23 & 1 \\
\hline
\end{tabular}

*Valores correspondentes à frequência total = percentual de respostas para cada item e respectivas opções.

A Tabela 3 apresenta os dados a respeito das perspectivas dos participantes do estudo quanto aos direitos das pessoas que têm problemas com drogas. Todos os itens mais elevados dos escores apresentados evidenciam falta de respeito aos usuários, em geral, pela polícia $(78 \%)$, pelo governo (62\%), pela justiça (55\%) e pelo sistema de saúde
(43\%). Embora com escores mais baixos, os participantes que assinalaram o sistema de saúde relataram situações que variaram de preconceito, formas de clientelismo até negligência e infração à norma legal no atendimento a usuários de drogas ilícitas, particularmente em unidades hospitalares de emergência.

Tabela 3 - Opinião sobre o respeito aos direitos das pessoas que têm problemas com drogas ilícitas*

\begin{tabular}{|c|c|c|c|c|c|}
\hline Domínio: leis e políticas & $\begin{array}{c}\text { Concorda } \\
\text { totalmente = } 1\end{array}$ & $\begin{array}{c}\text { Concorda } \\
\text { parcialmente }=2\end{array}$ & $\begin{array}{c}\text { Discorda } \\
\text { parcialmente }=3\end{array}$ & $\begin{array}{c}\text { Discorda } \\
\text { totalmente }=4\end{array}$ & Não sei $=8$ \\
\hline 1. Pela polícia & 2 & 10 & 10 & 78 & 0 \\
\hline 2. Pelo governo & 2 & 14 & 20 & 62 & 2 \\
\hline 3. Pelo sistema de saúde & 9 & 32 & 12 & 43 & 4 \\
\hline 4. Pela justiça & 6 & 18 & 19 & 55 & 2 \\
\hline
\end{tabular}

*Valores correspondentes à frequência total = percentual de respostas para cada item e respectivas opções.

Na Tabela 4 estão apresentadas as opiniões dos participantes sobre como são e como deveriam ser as leis e políticas relacionadas às drogas ilícitas, no Brasil. As respostas ficaram distribuídas em 31\% que deveriam mudar ou sofrer ajustes, $28 \%$ que deveriam ser mais punitivas, $21 \%$ que deveriam ser menos punitivas e $16 \%$ que deveriam ser mantidas como estão. Portanto, em $80 \%$ das perspectivas dos entrevistados na zona oeste do Rio de Janeiro, o sistema legal e de políticas públicas, relacionados às drogas ilícitas, seria beneficiado por algum tipo de revisão ou ajuste.

Tabela 4 - Opinião sobre as leis e políticas relacionadas às drogas ilícitas no Brasil

\begin{tabular}{lc}
\hline \multicolumn{1}{c}{ Domínio: leis e políticas } & $\begin{array}{c}\text { Total de } \\
\text { respostas } \\
\text { assinaladas }\end{array}$ \\
\hline 1. As leis e políticas devem continuar como estão & 16 \\
2. As leis e políticas deveriam punir mais as & 28 \\
pessoas (ser mais punitivas) & \\
3. As leis e políticas deveriam punir menos as & 21 \\
pessoas (ser menos punitivas) & 31 \\
4. Deveriam mudar. Deveria haver ajustes/revisão & 4 \\
5. Não sei & \\
\hline
\end{tabular}

\section{DISCUSSÃO}

No transcorrer das entrevistas quantitativas, várias anotações de detalhamento das respostas dos participantes foram registradas no rodapé das questões. Ao se cruzar tais infomações adicionais de ordem qualitativa, verificou-se que se tratava de ilustrações e explicações do porquê de suas escolhas e respostas nas escalas tipo Likert, apresentadas no artigo em tela.

Em especial, com relação às respostas sintetizadas na Tabela 2, ocorreram relatos de que usuários jovens, presos por porte de reduzida quantidade de droga para consumo próprio, foram alocados em ambiente carcerário compartilhado com traficantes e pessoas com sérios comprometimentos de conduta. Conduta que, na opinião dos participantes, não foi um facilitador de recuperação ou tratamento, pelo contrário ${ }^{(13)}$. Outra ilustração frequente foi a escassa oferta de serviços de saúde específicos, reiterando as respostas quantitativas relacionadas a respeito dos direitos das pessoas que têm problemas com as drogas ilícitas, uma vez que, mesmo quando 
procuram ajuda especializada, os usuários não a encontram no momento da busca. Também foram relatados casos de usuários não serem tratados adequadamente, quando atendidos em unidades hospitalares de emergência, ao informar aos profissionais $^{(14-15)}$ a dependência química de drogas ilícitas.

Por serem familiares e pessoas próximas a usuários, os participantes detinham vasto conhecimento das leis e políticas públicas relacionadas às drogas ilícitas, no Brasil. Na verdade, devido à proximidade afetiva da comunidade e de mais de um usuário, a grande maioria dos participantes já havia passado por ou observado alguma situação em que as leis e políticas foram inadequadas ${ }^{(13,16-18)}$.

Esta seção do estudo multicêntrico reiterou a importância da voz das pessoas que vivenciaram e sofreram junto à sua comunidade e seus residentes usuários, pois revelaram aspectos até então não documentados e publicados. Todavia, os resultados também desafiam os pesquisadores a expandir e aprofundar o tema - em estudos que não sejam apenas críticos, mas que resultem em recomendações e expansão do estudo para coletas-intervenção.

Embora de riqueza ímpar no que se propôs conhecer em cada cenário, no Brasil, na América Latina e Canadá, o estudo apresenta limitações. Logisticamente, o recrutamento de participantes foi difícil, o que demandou muito mais tempo do que o esperado para atingir o número pré-determinado de 100 participantes na comunidade localizada na zona oeste do Rio de Janeiro. Devido à extensão do instrumento quantitativo da pesquisa, as limitações do estudo incluíram a duração das entrevistas quantitativas (cerca de duas horas em média), uma amostragem não probabilística e pequena para o número de variáveis, o que não permite tratamento estatístico inferencial. Em conclusão, na verdade, as próprias limitações de um estudo representa um de seus aspectos mais fortes, pois apontam novos direcionamentos a seguir, lacunas a preencher, estudos subsequentes a serem implementados. Se, por um lado, o estudo não respondeu extensivamente a todas as dúvidas, certamente gerou inquietações e vontade de realizar estudos interventivos clínicos, sociais e políticos que tenham como objetivo dar retorno concreto para a comunidade e para os participantes de projetos de pesquisa - ainda em seu tempo de vida o que deveria representar obrigatoriedade para pesquisadores das ciências da saúde, ciências sociais e humanas que investigam drogas ilícitas.

\section{AGRADECIMENTOS}

Esta pesquisa foi realizada com o apoio, assessoria e patrocínio do governo do Canadá, da Organização dos Estados Americanos (OEA), da Comissão Inter-Americana para o Controle e Abuso de Drogas (CICAD), e do Centro de Drogas e Saúde Mental (CAMH), Canadá. Agradecemos a colaboração de outros colegas que contribuíram de forma direta ou indireta na realização deste estudo.

\section{REFERÊNCIAS}

1. World Health Organization (USA). World Health Report. Working Together for Health. Geneva: WHO; 2006.

2. CICAD/OEA. Mecanismos de Avaliação Multilaterais, 2003: Implementação do mecanismo de avaliação multilateral da Comissão Interamericana para o Controle do Abuso de Drogas. Washington, DC: OEA; 2003. [Comissão Interamericana para o Controle do Abuso de Drogas (CICAD), documento $\mathrm{cp} /$ doc.3711/03].

3. CICAD/OEA. Mecanismos de Avaliação Multilaterais, 2004: Projeto de resolução "Observações e recomendações sobre o relatório anual da Comissão Interamericana para o Controle do Abuso de Drogas (CICAD)" (Apresentado pela Delegação do Canadá) CP/CG-1585/04. Washington, DC: OEA; 2004

4. CICAD/OEA. Mecanismos de Avaliação Multilaterais, 2005: Projeto de resolução: Mecanismo de Avaliação Multilateral (MAM) da Comissão Interamericana para o
Controle do Abuso de Drogas: Relatório de sua Terceira Rodada de Avaliação CP/CG-1635/05 rev.1. Washington, DC: OEA; 2005.

5. Wright MGM, Caufield C, Gray G, Olson J. International research capacity-building programs for nurses to study the drug phenomenon in Latin America: challenges and perspectives. Rev Latino-am Enfermagem. 2005; 13(n. espe 2): 1095-101.

6. United Nations. United Nations Office on Drugs and Crime. World Drug Report. United Nations Publication Sales No. E.04.XI.16. 2006; 1:1-214.

7. Creswell JW. Research design: qualitative, quantitative and mixed method approaches. 2nd ed. Thousand Oaks (CA): Sage; 2003.

8. Goering PL, Streiner DL. Reconcilable differences: the marriage of qualitative and quantitative methods. Can J Psychiatr 1996; 41(1):491-7.

09. Browne K. Snowball sampling: using social networks to research non-heterosexual women. Int J Soc Res Methodol 
$2005 ; 8(1): 47-60$.

10. Charmaz K. Constructing Grounded Theory. Thousand Oaks (CA): SAGE; 2006.

11. Morse JM. Principles of sampling. In: Morse JM, editor. Qualitative nursing research: a contemporary dialogue. Newbury Park: SAGE; 1995.

12. Morse JM, Barrett M, Mayan M, Olson K, Spiers J. Verification strategies for establishing reliability and validity in qualitative research [online]. 2002. Available: http:// www.ualberta.ca/ ijqm/

13. Reghelin EM. Considerações político-criminais sobre o uso de drogas na nova legislação penal brasileira. Instituto Brasileiro de Ciências Criminais, São Paulo. Rev Bras Ciênc Criminais 2007 janeiro/fevereiro; 15(64):57-77.

14. Rezende MM. Uma provável caracterização do profissional de saúde mental que atende dependentes de drogas no Vale do Paraíba. Rev Latino-am Psicopatol Fundament. 2002; 6(1):100-8. Disponível em: http://www.fundamentalpsychop athology.org/art/mar3/7.pdf

15. Cruz MS, Silva JF Filho. A formação de profissionais para a assistência de usuários de drogas e a constituição de um novo habitus de cuidado. J Bras Psiquiatr. 2005; 54(2):1206. 16. Giacomolli NJ. Análise crítica da problemática das drogas e a lei 11.343/2006. Instituto Brasileiro de Ciências Criminais. Rev Bras Ciênc Criminais. 2008 março/abril; 16(71)181-204. 17. Gomes LF. Nova lei de drogas: descriminalização da posse de drogas para consumo pessoal. Jus Navigandi novembro de 2006; 11:1236. Disponível em: http://jus2.uol.com.br/ doutrina/texto. asp?id $=9180$

18. Karam ML. Drogas e redução de danos. Instituto Brasileiro de Ciências Criminais, São Paulo. Rev Bras Ciênc Criminais. 2007 janeiro/fevereiro; 15(64):128-44. 